patients admitted to the AICU, 30.5\% died, $69.0 \%$ were transferred to another unit, and $0.5 \%$ were discharged.

Conclusion The findings showed high compliance of AST at admission but low compliance at discharge, which needs further enforcement. Although the acquisition of CRKP was only $3.4 \%$, it represents adding almost double (1.8) new cases of CRKP to the admission level. Future research is required to assess the impact of AST on the burden of healthcare-associated infections.

\section{EVALUATION OF ORAL ANTICANCER MEDICATION HANDLING, STORAGE, AND DISPOSAL PRACTICES AMONG CANCER PATIENTS AND THEIR CAREGIVERS IN THE HOME SETTING AT PRINCESS NORAH ONCOLOGY CENTER}

Ashwag A Algethami, Majed A Alshamrani, Atika M AlHarbi, Aeshah A AlAzmi, Mansoor A Khan. Pharmaceutical Care Services Department, Ministry of National Guard Health Affairs, King Abdulaziz Medical City Jeddah, Saudi Arabia

\subsection{6/bmjoq-2019-PSF.6}

Background Oral chemotherapeutic agents are hazardous drugs that are commonly prescribed for a variety of indications. Using oral anticancer medications may ensure better therapy adherence, but there is not much knowledge about the safety and risks of accidental exposure with subsequent fatalities. Unintentional exposure can take place at different phases of treatment by splitting, crushing, transporting, unpacking, storage, and disposal. Although there are several well-established guidelines for safe handling of parenteral chemotherapy in the healthcare setting, there are few recommendations concerning proper handling of oral chemotherapy at home for patient and environmental safety. This survey aimed to evaluate the handling, storage, and disposal practices of oral anticancer medications among cancer patients and their caregivers in the home setting.

Methods A questionnaire-based cross-sectional study was done in all adult cancer patients/patient caregivers receiving oral anticancer medications and visiting the oncology outpatient pharmacy. Oncology pharmacists interviewed patients after obtaining consent. Survey responses were analysed using descriptive statistics.

Results A total of 201 participants agreed to be interviewed, of whom most were female (67\%). Nearly $44 \%$ of participants were aged between 40 and 60 years. The majority of participants were educated (157 responses, 78\%) while only $44(22 \%)$ had never been to school. The top five oral anticancer medications taken by patients were tamoxifen, capecitabine, letrozole, dasatinib, and imatinib. All participants reported that medications were kept away from children and pets (100\%). $196(97.5 \%)$ patients responded that the medication was stored away from extreme heat, cold, and humidity. $195(97 \%)$ patients reported keeping their medications in the original container. Hand washing and wearing gloves were not a consistent practice among patients and caregivers. Among all participants, only nine (4.5\%) reported 'always' wearing gloves; 48 (24\%) reported 'always' washing hands after handling anticancer medications. Patients and caregivers reported that they had been informed about safe handling and storage by their physician (47\%) and pharmacist $(30 \%)$, while $40 \%$ of them had not been informed. In terms of disposal practice, more than half $(66 \%)$ of patients had not had unused or expired medications, $29 \%$ of patients dispose of them in the trash, and $27 \%$ return them to the pharmacy or doctor's office.

Conclusion Our findings, while preliminary, suggest that patients' and caregivers' awareness regarding handling practices are inconsistent with the published recommendations. Appropriate and comprehensive education is needed to mitigate exposure risk to these agents in the home setting.

\section{REDUCE MEDICATION ADMINISTRATION DELAY IN NEONATAL INTENSIVE CARE UNIT}

Noura Alshami, Amerah NAl Saleh, Amjed Abu Alburak, Regina Manlulu, Saif Al Saif, Mark Anthony Castro, Eden Grace Abainza, Suzan Ziyad Omran, Yvonne Marlene Makins, Mary Ann Rivera, Flordelisa Ramos. Neonatal Intensive Care Unit, King Abdulaziz Medical City Riyadh, Ministry of National Guard Health Affairs

\subsection{6/bmjoq-2019-PSF.7}

Background Medication errors are the most common medical errors experienced by patients. Neonates are even more vulnerable to medication errors because of drug dosing that is influenced by weight, gestational age, and postnatal age. Data obtained from the safety reporting system related to medication and fluids events in the neonatal intensive care unit (NICU) from January to August 2017 indicated a high rate of near-misses and errors reaching patients. The top five specific event types related to medication and fluids events were, in the following order: delay, incorrect dose, incorrect frequency, medication packaging issues, and dose omitted.

Methods In September 2017, a project team was formed including members from the following disciplines: neonatology, neonatal nursing, pharmacy, medication safety program, and quality department. Data obtained from the safety reporting system were examined and analyzed. After thorough analysis of the data, the project team agreed to tackle the delay in STAT medication administration in the first cycle of the project. The team also completed a process map and cause and effect diagram to understand the root causes of the delay. The average time required for a STAT medication or fluid to be administered to the NICU patient was 3 hours, and the delay in STAT medication was mainly related to septic shock and suspected sepsis management. To reduce the delay in STAT medication administration, the NICU must be provided with a floor stock of pre-mixed antibiotics that is readily available for administration when required. A standardized order set for septic shock doses was programed in the BestCare System to reduce the time and effort required in generating orders. All the stakeholders involved in the process were made aware of the new changes and a departmental policy was created to guide the staff practice.

Results After the implementation of the pre-mixed antibiotic floor stock and pre-set orders, the turnaround time for administration of STAT medication in the NICU reduced from 3 hours to 1 hour, and there was a $60 \%$ reduction in the delay in medication administration events.

Conclusion The new changes in the process impacted positively on patient safety and prevention of harm. They reduced time and redundancy in the previous process. The changes also improved staff satisfaction and reduced the tension that used to result from the frequent communication and followups involved until the appropriate medication was made available for administration. 